\title{
ROLE OF SERUM UROMODULIN IN THE EARLY DIAGNOSIS OF CHRONIC KIDNEY DISEASE
}

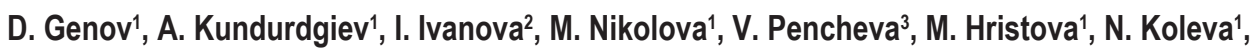 \\ A. Kostadinova ${ }^{1}$, Ts. Vutova ${ }^{1}$ \\ ${ }^{1}$ Clinic of Nephrology, University Hospital "Sv. Ivan Rilski”, Medical University - Sofia, Bulgaria \\ ${ }^{2}$ Clinical Laboratory, University Hospital "Sv. Ivan Rilski" - Sofia, Bulgaria \\ ${ }^{3}$ Clinic of Pulmology, Department of Internal Medicine, University Hospital "Alexandrovska", \\ Medical University - Sofia, Bulgaria
}

\begin{abstract}
Background: The diagnosis of chronic kidney disease (CKD) is usually delayed, when significant functional renal impairment has already occurred. The diagnosis is complex and clinical and laboratory investigations play a crucial role. There are well-established markers of CKD - serum creatinine and cystatin C. However, the search for new reliable biomarkers that aid in the assessment of kidney function and predict the evolution of the disease is still in progress. Objective: To investigate the role of serum uromodulin (sUmod) as a marker for early diagnosis of renal impairment in patients with CKD. Materials and Methods: We investigated 70 patients, 28 male and 42 female, mean age $56.53 \pm 11.753$, with CKD in a prospective observational study. All patients were admitted to the Clinic of Nephrology at the "St. Ivan Rilski" University Hospital between April and November 2019. After obtaining written informed consent, laboratory blood and urine tests, abdominal ultrasound and sUmod investigations were performed in all patients. Results: Plasma uromodulin levels showed decrease with the increasing of the severity of renal impairment. sUmod displayed inverse correlation with serum creatinine $(r=-0.467, p<0.0001)$, cystatin $C(r=-0.430, p<0.0001)$ and urea $(r=-0.495, p<0.0001)$ and a positive correlation with eGFR ( $r=0.628, p<0.0001)$. Conclusion: The results of our study show that sUmod levels significantly correlate with all established laboratory parameters used for the evaluation of renal impairment. It can be used as a potential early biomarker for CKD diagnosis.
\end{abstract}

Key words: serum uromodulin, chronic kidney disease, biomarker, serum creatinine, cystatin C

Corresponding author: Diyan Genov, University Hospital "Sv. Ivan Rilski" 15 Acad. Ivan Geshov blvd., Medical University, Sofia, Bulgaria, Tel: ++359 887837107, E-mail: dian.genov@abv.bg

RECEIVED: 24 March 2020, ACCEPTED: 9 July 2020

\section{INTRODUCTION}

$\mathrm{T}$ he diagnosis of chronic kidney disease (CKD) is usually belated, when significant functional renal impairment has already occurred, due to its nonspecific symptoms. CKD encompasses five stages, and progresses slowly from mild to moderate and to severe reduction in glomerular filtration. The risk for the development of CKD increases with age. The diagnosis is complex and is based on laboratory and instrumental tests and kidney biopsy [1-5].

The clinical-laboratory investigations have crucial role for the diagnosis of CKD, because they provide fast, easy, and relatively inexpensive tests with 
discriminative results. Although there are wellestablished markers, such as serum creatinine $(\mathrm{SCr})$ and cystatin $\mathrm{C}$ (CysC), the search for new reliable biomarkers that would aid the assessment of kidney function and the prediction of the evolution of CKD is still in progress [6-7]. Several new markers for the evaluation of renal function recently have been introduced for scientific purposes, and some of them are being used routinely. Uromodulin, also known as Tamm-Horsfall protein, is a promising marker for the diagnosis of renal damage, but its clinical significance needs more scientific evaluation [811]. Uromodulin is produced almost entirely by the kidney and is the most abundant protein excreted with the normal urine. In the past, urinary uromodulin has been studied more extensively, but the role of serum uromodulin (sUmod) remains unclear. sUmod is a stable monomeric antigen and it seems a more reliable marker than urinary $[12,13]$.

The aim of the study was to investigate the role of sUmod as a marker for early diagnosis of renal impairment in patients with chronic renal diseases.

\section{MATERIAL AND METHODS}

Overall 70 patients ( 28 male and 42 female, mean age $56.53 \pm 11.753$ ) diagnosed with CKD were included in this prospective observational study. All subjects were admitted to the Clinic of Nephrology at the University Hospital St. Ivan Rilski" between April and November 2019. The study was approved by the local ethics committee of Medical University, Sofia, Bulgaria, in adherence with the declaration of Helsinki. Prior to the inclusion in the study, written informed consent was obtained from all studied subjects. All the patients were $>18$ years of age. Arterial hypertension had $64 / 70(91.43 \%)$ and $39 / 70(55.71 \%)$ had diabetes, with optimal glycaemic control at the time of the investigation (in the cases with diabetes) defined as $\mathrm{HbA} 1 \mathrm{c}<7 \%$. Patients with acute or chronic infections, mental disorders, organ transplant recipients, proven oncological diseases, prolonged use (over the last six months) and during the study of non-steroidal antiinflammatory drugs, corticosteroids, hormonal preparations or antioxidants were excluded from the study. Furthermore, patients with symptomatic or asymptomatic urinary tract infection, defined as detection of leucocytes and/or bacteria in the urinary sediment at the time of enrollment, were excluded.

Medical history and physical examination were performed in all participants. Haematological and biochemical blood tests and urine tests were performed and 24-hours proteinuria was calculated, using standard methods. Estimated glomerular filtration rate (eGFR) was evaluated using the abbreviated Modification of Diet in Renal Disease (MDRD) equation: eGFR (mL/ $\left.\mathrm{min} / 1.73 \mathrm{~m}^{2}\right)=175 \times \mathrm{SCr}-1.154 \times$ Age $-0.203 \times 0.742$ (if woman) $\times 1.21$ (if black) $[14,15]$.

CysC was measured usingh Human ELISA Cystatin $C$ kit (Biovendor Research and Diagnostic Products, Cat. No: RD191009100, Germany) by the particle enhanced immune nephelometric method (normal range $0.50-0.96 \mathrm{mg} / \mathrm{L}$ ). In all patients abdominal ultrasound with duplex Doppler examination of the kidneys was performed.

The investigation of sUmod was performed with uromodulin-ELISA kit (Euroimmun AG, Lübeck, Germany). Overall $5 \mathrm{~mL}$ venous blood was collected from each participant. Then the plasma was prepared by centrifugation at $2000 \times \mathrm{g}$ for $30 \mathrm{~min}$ at $4^{\circ} \mathrm{C}$. All plasma samples were stored at $-80^{\circ} \mathrm{C}$ before measurements were performed. A 96-well plate was coated with uromodulin in advance and blocked to reduce non-specific binding. Plasma samples were diluted 1:101 using dilution buffer. A total of $100 \mu \mathrm{L}$ of calibrators, controls, or diluted samples were pipetted into coated wells of the microtiter plate, subsequently $100 \mu \mathrm{L}$ of biotinylated detection antibody (final concentration $50 \mathrm{ng} / \mathrm{mL}$ ) were added. The microtiter plate was covered with foil and incubated for 120 minutes at 450 rotations per minute $(\mathrm{rpm})$ and room temperature $\left(+18^{\circ} \mathrm{C}\right.$ to $\left.+25^{\circ} \mathrm{C}\right)$ on a rotary shaker. After 2 hours the microtiter plate was washed 3 times using $300 \mu \mathrm{L}$ washing buffer, then the wells were tapped gently. A total of $100 \mu \mathrm{L}$ of streptavidin-peroxidase (final concentration $67 \mathrm{ng} / \mathrm{mL}$ ) were pipetted into each well followed by another incubation for 30 minutes at $450 \mathrm{rpm}$. Subsequently, the streptavidin-peroxidase was soaked and the microtiter plate washed 3 times with $300 \mu \mathrm{L}$ of washing buffer. Consequently, $100 \mu \mathrm{L}$ of substrate solution (containing the chromogen tetramethylbenzidin and hydrogen peroxide as the substrate for streptavidin-peroxidase) were pipetted into each well. The microtiter plate was incubated in the dark for 15 minutes at room temperature. The reaction was terminated by adding $100 \mu \mathrm{L}$ of stop solution. This causes a color change from blue to yellow. Finally, the substrate solution was measured using a photometer at a wavelength of $450 \mathrm{~nm}$ and a reference wavelength between $620 \mathrm{~nm}$ and $650 \mathrm{~nm}$. For this assay the mean linearity recovery was $97 \%$ (83\%-107\% at $59-397 \mathrm{ng} / \mathrm{mL})$; intra-assay precision $-1.8-3.2 \%$ (at 30-214 $\mathrm{ng} / \mathrm{mL}$ ), inter-assay precision $6.6 \%$ to $7.8 \%$ (at $35-228 \mathrm{ng} / \mathrm{mL}$ ), and inter-lot precision $-7.2 \%$ to $10.1 \%$ (at $37-227 \mathrm{ng} / \mathrm{mL}$ ). The lower detection limit of the uromodulin ELISA was $2.0 \mathrm{ng} / \mathrm{mL}$.

The statistical analysis was performed using SPSS vs.19 for Windows. The statistical analyses included a variational analysis of the quantitative 
variables - median, standard deviation, standard error of the median and $95 \%$ confidence interval of the median and frequency analysisof qualitative variables. Kolmogorov-Smirnov, Mann-Whitney and Kruskal-Wallis Tests were used for testing the normality of distribution of the quantitative variables. Correlations were tested using the Spearman's tests. All data are presented as mean values \pm SD or as numbers, unless specified otherwise. All values of $P \leq 0.05$ were considered statistically significant.

\section{RESULTS}

The study included 70 patients with chronic renal impairment, 28 male and 42 female, mean age of the patients was $56.53 \pm 11.753$ years. The maleto-female ratio was $28 / 42(40.0 \%$ men and $60.0 \%$ women). The main comorbidities were arterial hypertension - in $64(91.43 \%)$ and diabetes mellitus - in $39(55.71 \%)$. The main clinical and laboratory data of patients are presented in Table 1.

Table 1. Characteristics of the studied patients

\begin{tabular}{|l|l|}
\hline Parameter & Mean \pm SD \\
\hline Weight $(\mathrm{kg})$ & $79.99 \pm 19.119$ \\
\hline Height $(\mathrm{cm})$ & $167.51 \pm 8.893$ \\
\hline Body Mass Index & $28.354 \pm 5.844$ \\
\hline Systolic blood pressure $(\mathrm{mm} \mathrm{Hg})$ & $136.79 \pm 15.972$ \\
\hline Diastolic blood pressure $(\mathrm{mm} \mathrm{Hg})$ & $87.07 \pm 10.197$ \\
\hline Uric acid $(\mu \mathrm{mol} / \mathrm{L})$ & $383.06 \pm 112.256$ \\
\hline SCr $(\mu \mathrm{mol} / \mathrm{L})$ & $191.17 \pm 156.538$ \\
\hline Urea $(\mathrm{mmol} / \mathrm{L})$ & $12.136 \pm 7.669$ \\
\hline eGFR $\left(\mathrm{mL} / \mathrm{min} / 1.73 \mathrm{~m}{ }^{2}\right)$ & $40.83 \pm 26.019$ \\
\hline CysC $(\mathrm{mg} / \mathrm{L})$ & $2.549 \pm 1.636$ \\
\hline sUmod $(\mathrm{ng} / \mathrm{ml})$ & $123.237 \pm 128.611$ \\
\hline
\end{tabular}

SCR = serum creatinine, eGFR = estimated glomerular filtration rate $(\mathrm{MDRD}), \mathrm{Cys} \mathrm{C}=\mathrm{Cystatin} \mathrm{C}, \mathrm{sUmod}=$ serum uromodulin.
The patients were divided into 5 groups according to the KDIGO Recommendations: CKD stage I (eGFR > $90 \mathrm{~mL} / \mathrm{min} / 1.73 \mathrm{~m}^{2}$ ) - 11; CKD stage II (eGFR: 60 to $89 \mathrm{~mL} / \mathrm{min} / 1.73 \mathrm{~m}^{2}$ ) - 17; stage IIla (eGFR: 45 to 59 $\mathrm{mL} / \mathrm{min} / 1.73 \mathrm{~m}^{2}$ ) -6 ; CKD stage IIIb (eGFR: 30 to 44 $\mathrm{mL} / \mathrm{min} / 1.73 \mathrm{~m}^{2}$ ) - 13; CKD stage IV (eGFR: 15 to 29 $\left.\mathrm{mL} / \mathrm{min} / 1.73 \mathrm{~m}^{2}\right)$ - 13; CKD stage $\mathrm{V}($ eGFR $<15 \mathrm{~mL} /$ $\left.\mathrm{min} / 1.73 \mathrm{~m}^{2}\right)-10$. The mean levels of sUmod, CysC, $\mathrm{SCr}$ and urea in the different groups are presented in Table 2.

The correlation analysis revealed a negative correlation between sUmod and $\mathrm{SCr}(r=[-0.467]$, $p<0.0001)$, CysC $(r=[-0.430], p<0.0001)$ and urea $(r=[-0.495], p<0.0001)$, and a positive relation with $\operatorname{eGFR}(r=0.628, p<0.0001)$.

\section{DISCUSSION}

$\mathrm{SCr}$ and urea levels, CysC and eGFR are wellestablished markers for the diagnosis of CKD. Yet, these markers seem to be insufficient and sometimes inaccurate for early diagnosis [9]. Therefore, new validated biomarkers are needed for a better assessment of CKD and its progression. We investigate the role of sUmod as a marker for early diagnosis of renal impairment in CKD. In our study the mean plasma uromodulin level was $123.2367 \mathrm{ng} / \mathrm{mL}$ for all CKD stages combined (from 2.64 to 709.64 $\mathrm{ng} / \mathrm{mL}$ ) and sUmod decreased with the increasing of the degree of renal impairment. The lower values were measured in higher stages of CKD. Similar results were obtained by Scherberich et al. [16], who reported similar sUmod levels - from 1 to $667 \mathrm{ng} /$ $\mathrm{mL}$ with a median of $85 \mathrm{ng} / \mathrm{mL}$. In their study sUmod decreased with progressive CKD as well [16].

Previous studies found that sUmod concentrations were significantly lower among patients with CKD than in controls and displayed inverse correlation with creatinine [17-19]. Our results confirm once

Table 2. Mean levels of sUmod, CysC, serum creatinine and urea in different CKD groups

\begin{tabular}{|c|c|c|c|c|}
\hline CKD stage & sUmod (ng/ml) & CysC (mg/L) & $\mathrm{SCr}(\mu \mathrm{mol} / \mathrm{L})$ & Urea (mmol/L) \\
\hline$I(n=11)$ & $228.008 \pm 176.267$ & $1134.337 \pm 384.683$ & $74.00 \pm 11.636$ & $4.862 \pm 1.809$ \\
\hline$\|(n=17)$ & $200.889 \pm 117.161$ & $1502.398 \pm 759.831$ & $78.41 \pm 9.428$ & $5.297 \pm 1.244$ \\
\hline IIla $(n=6)$ & $109.015 \pm 48.434$ & $2165.468 \pm 758.963$ & $118.33 \pm 18.118$ & $9.100 \pm 4.619$ \\
\hline IIlb $(n=13)$ & $74.464 \pm 50.127$ & $3153.244 \pm 2028.953$ & $157.85 \pm 33.148$ & $12.070 \pm 2.364$ \\
\hline IV $(n=13)$ & $45.112 \pm 20.547$ & $3384.859 \pm 965.872$ & $264.62 \pm 50.791$ & $20.54 \pm 5.043$ \\
\hline$V(n=10)$ & $42.439 \pm 35.050$ & $4129.085 \pm 1812.850$ & $503.30 \pm 143.146$ & $24.870 \pm 6.666$ \\
\hline
\end{tabular}


again that plasma uromodulin expression significantly negatively correlates with $\mathrm{SCr}$ levels $(r=[-0.467])$.

Steubl et al. tested plasma uromodulin in 426 individuals of whom 71 were CKD stage 0 and 355 had CKD and found that uromodulin correlated well with CysC ( $r=[-0.79])$ [19]. Similar results were reported by other authors $[16,18]$. In our study sUmod concentrations are strongly negatively correlated with renal CysC ( $r=[-0.353])$.

Risch et al. [18] tested 289 participants with different stages of kidney function (i.e., cystatin C-based 2012-CKD-EPI eGFRCysC $>90 \mathrm{~mL} / \mathrm{min} / 1.73 \mathrm{~m}^{2}, 60$ $89 \mathrm{~mL} / \mathrm{min} / 1.73 \mathrm{~m}^{2}, 45-59 \mathrm{~mL} / \mathrm{min} / 1.73 \mathrm{~m}^{2}$, and $<45$ $\mathrm{mL} / \mathrm{min} / 1.73 \mathrm{~m}^{2}$ ). They found significant differences in serum uromodulin among the four groups according to different kidney function stages $(p<0.001)$. sUmod displayed inverse relationships with urea $(r=-0.30)$ [18]. Scherberich et al. [16] and Steubl et al. [19] also found significant negative correlation between sUmod and BUN ( $r=-0.645$ and -0.72 respectively). Our results are similar and sUmod concentrations show strong negative correlation with urea $(r=$ [-0.495]).

Lv et al. investigated 2652 CKD patients and found a positive correlation between sUmod and eGFR in multivariable linear correlation analysis $(r=0.68$, $p<0.001$ ) [10]. Similar significant relation have been reported by Steubl et al. [19] and Leiherer et al. [20] ( $r=0.80$ and $r=0.242$ respectively). Our results show statistically significant correlation between sUmod and eGFR $(r=0.628)$.

\section{STUDY LIMITATIONS}

One limitation of our study is the relatively small sample size and the single-center recruitment. The number of patients in the different CKD groups is not sufficient for in-depth statistical analysis. Large cohort studies in the future are needed to confirm our findings.

\section{CONCLUSIONS}

Our study shows that sUmod levels significantly correlate with all well-known and already established laboratory parameters used for evaluation of renal impairment. Our findings support the use of sUmod as a potential early biomarker for the diagnosis of CKD.

Disclosure summary: The authors have nothing to disclose.

\section{Acknowledgement}

This project is sponsored by MU-Sofia, as part of Grant D-117/2019.

\section{REFERENCES}

1. Couser WG, Remuzzi G, Mendis S et al. The contribution of chronic kidney disease to the global burden of major noncommunicable diseases. Kidney Int 2011; 80: 1258-1270.

2. National Kidney F. KDIGO clinical practice guidelines for evaluation and management of chronic kidney disease. Kidney Int Suppl 2013;3(1):9л

3. Arnold R, Issar T, Krishnan AV et al. Neurological complications in chronic kidney disease. JRSM Cardiovasc Dis 2016;5: 204.

4. Brachemi S, Bollée G. Renal biopsy practice: What is the gold standard? World J Nephrol 2014;3(4):287-94.

5. Visconti L, Cernaro V, Ricciardi CA et al. Renal biopsy: still a landmark for the nephrologist. World J Nephrol 2016; 5(4):321-7.

6. Rysz J, Gluba-Brzózka A, Franczyk B et al. Novel Biomarkers in the Diagnosis of Chronic Kidney Disease and the Prediction of Its Outcome. Int J Mol Sci 2017 Aug 4;18(8). pii: E1702.

7. Gentile G, Remuzzi G. Novel biomarkers for renal diseases? None for the moment (but one). J Biomol Screen 2016;21(7):655-70.

8. Tan $F$, Zeng $Y$, Yan $L$ et al. Low plasma uromodulin is a predictor of early stage chronic kidney disease progression. Int $\mathrm{J}$ Clin Exp Med 2017; 10:8055-8059.

9. Devuyst O, Olinger E, Rampoldi L. Uromodulin: from physiology to rare and complex kidney disorders. Nat Rev Nephrol 2017;13(9):525-44.

10. Lv L, Wang J, Gao B et al. Serum uromodulin and progression of kidney disease in patients with chronic kidney disease. $J$ Transl Med 2018; 16(1) :316.

11. Garimella PS, Sarnak MJ. Uromodulin in kidney health and disease. Curr Opin Nephrol Hypertens 2017;26(2):136-142.

12. Garimella PS, Biggs $M L$, Katz $R$ et al. Urinary uromodulin, kidney function, and cardio-vascular disease in elderly adults. Kidney Int 2015;88:1126-34.

13. Youhanna S, Weber J, Beaujean $\mathrm{V}$ et al. Determination of uromodulin in human urine. Influence of storage and processing. Nephrol Dial Transplant 2014;29:136-45.

14. Inker $\mathrm{LA}$, Schmid $\mathrm{CH}$, Tighiouart $\mathrm{H}$ et al. Estimating glomerular filtration rate from serum creatinine and cystatin $\mathrm{C}$. N Engl J Med 2012; 367:20-29.

15. Levey AS, Coresh J, Greene T et al. Using standardized serum creatinine values in the modification of diet in renal disease study equation for estimating glomerular filtration rate. Ann Intern Med 2006;145(4):247-54.

16. Scherberich JE, Gruber R, Nockher WA et al. Serum uromodulin-a marker of kidney function and renal parenchymal integrity. Nephrol Dial Transplant 2017. doi:10.1093/ndt/ gfw422.

17. Fedak D, Kuźniewski M, Fugiel A et al. Serum uromodulin concentrations correlate with glomerular filtration rate in patients with chronic kidney disease. Pol Arch Med Wewn 2016;126(12):995-1004.

18. Risch L, Lhotta K, Meier D et al. The serum uromodulin level is associated with kidney function. Clinical Chemistry and Laboratory Medicine 2014;52(12):1755-1761.

19. Steubl D, Block M, Herbst V et al. Plasma Uromodulin Correlates With Kidney Function and Identifies Early Stages in Chronic Kidney Disease Patients. Medicine (Baltimore) 2016;95(10):e3011.

20. Leiherer A, Muendlein A, Saely $\mathrm{CH}$ et al. The value of uromodulin as a new serum marker to predict decline in renal function. J Hypertens 2018;36(1):110-118. 